\title{
Cambios fisiológicos de la rotación de la marcha durante el desarrollo
}

\author{
ANGÉLICA IBÁÑEZ L. ${ }^{1}$, ALEJANDRO BAAR Z. ${ }^{1}$, NATALIA GANA A. ${ }^{2}$ \\ 1. Médico. Unidad de Ortopedia infantil. Departamento de Ortopedia y Traumatología. Pontificia Universidad Católica de Chile. \\ 2. Médico. Unidad de Investigación Clínica. Departamento de Ortopedia y Traumatología. Pontificia Universidad Católica de Chile.
}

\begin{abstract}
Physiological changes of walking rotation during development

Background: Feet rotation during walking is a frequent worrying motive. Most of the time, it corresponds to physiological conditions and variations within the normal range. Objective: Evaluate the factors involved in walking on healthy children, according to physical examination performed by Staheli method and its correlation with symptoms. Method: Evaluation of 610 healthy children at Santiago - Chile; 10 cases were excluded due to previous orthopedic pathology. A questionnaire was applied, which included perinatal factors, previous pathologies and lower limb related symptoms. An anthropometric evaluation and specific physical examination of lower limb rotations was also performed. The data was statistically analysed. Results: $58 \%$ of cases presented neutral walking, $23 \%$ of children had convergent walking and $19 \%$ presented divergent walking. There is a direct relationship between age and type of walking, being convergent walking most frequent at lower ages, due to bigger hip anteversion and greater internal tibial torsion. The plane foot and metatarsus adductus do not influence lower limb rotation. Obese children presented divergent walking most frequently. There are no significant differences in terms of lower limb pain and walking alterations. Conclusions: Walking has a known natural history, with a wide normality range and most of the time, it solves spontaneously without the need of specific therapy.

(Key words: Walking, development, plane foot, rotation, obesity).

Rev Chil Pediatr 2008; 79 (1): 45-49
\end{abstract}

\section{RESUMEN}

Introducción: La rotación de los pies al caminar es un motivo de preocupación y consulta frecuente. La gran mayoría de las veces corresponde a condiciones fisiológicas y variaciones dentro de lo normal, por lo que deben ser tratadas como tales. Objetivo: Evaluar tipo de marcha y factores involucrados en niños sanos, según el examen físico por método de Staheli y correlacionarlo con la presencia de síntomatología.

Trabajo recibido el 28 de septiembre de 2007, devuelto para corregir el 21 de noviembre de 2007, segunda versión el 26 de diciembre de 2007, aceptado para publicación el 16 de enero de 2008.

Correspondencia a:

Angélica Ibáñez L.

E-mail: maibanez@med.puc.cl 
Pacientes y Método: Evaluación de 610 niños sanos en Santiago de Chile. Se excluyeron 10 niños por patología ortopédica previa. Se aplicó encuesta que incluyó factores perinatales, patologías previas y síntomas de extremidades inferiores. Se realizó una evaluación antropométrica, examen físico general y dirigido a las rotaciones de las extremidades inferiores. Los datos se analizaron estadísticamente. Resultados: La mayoría presentó marcha en neutro: 58\%, seguido por la marcha convergente: 23\%, y por marcha divergente en 19\%. Se encontró una directa relación entre la edad y el tipo de marcha, siendo a menor edad más frecuente la marcha convergente, lo que tiene relación con la mayor antetorsión de las caderas y la mayor torsión tibial interna. El pie plano o metatarso varo no influyen en el tipo de marcha. Los pacientes obesos presentaron mayor prevalencia de marcha divergente. No hubo diferencias significativas respecto al dolor en extremidades inferiores ni alteraciones en la marcha. Se concluye que, en concordancia con la literatura, la marcha tiene una historia natural conocida, que el rango de normalidad es amplio, que la mayoría se resuelve espontáneamente y no requiere de tratamiento específico.

(Palabras clave: marcha, desarrollo, pie plano, metatarso varo, Staheli).

Rev Chil Pediatr 2008; 79 (1): 45-49

\section{Introducción}

La rotación de los pies en la marcha, es un motivo de consulta frecuente, tanto para el pediatra como para el traumatólogo infantil.

Es habitual que los padres se muestren preocupados por la capacidad de hacer una vida normal, las caídas frecuentes y el desempeño deportivo futuro del niño. Muchas veces buscan algún tipo de tratamiento, ya que existe la idea que el uso de plantillas, zapatos ortopédicos u otros artículos desrotadores, ayudarían a cambiar el tipo de marcha ${ }^{1-6}$.

Son conocidos los factores anatómicos que están involucrados en la rotación de los pies al caminar. Los que más influyen son la torsión del fémur proximal, la torsión de la tibia y la forma de los pies. Estas características anatómicas son evaluables en forma simple a través del examen físico con el método descrito por Staheli ${ }^{7-9}$.

La rotación de las caderas es signo de la torsión del fémur proximal. Es así como la rotación interna aumentada, indica una mayor anteversión femoral, y por el contrario la rotación externa indica el grado de retroversión femoral. La torsión de las tibias se mide a través del ángulo muslo-pie.

Durante el desarrollo, el tipo de marcha tiene una evolución variable y conocida ${ }^{10-13}$.

El enfoque organizado y metódico del examen físico nos va a permitir identificar la causa anatómica y descartar las condiciones patológicas que pudieran estar afectando la marcha.

En nuestro país no se han realizado hasta ahora estudios poblacionales analizando este tema.

Objetivo: Describir los perfiles rotacionales de marcha de los niños chilenos y las características anatómicas que influyen en ellos.

\section{Pacientes y Método}

A través de una campaña institucional, realizada en julio de 2004, fueron evaluados 610 niños entre 1 y 15 años de edad. Los niños se reclutaron a través de un llamado en la prensa para evaluar en forma gratuita del punto de vista ortopédico a niños sanos. Se citaron en nuestro consultorio institucional, donde se les aplicó una encuesta por enfermera universitaria, registrando antecedentes del embarazo, del parto, hitos del desarrollo psicomotor, problemas ortopédicos personales y familiares. Se preguntó específicamente respecto a presencia de dolor en extremidades inferiores, agrupándolos en 4 categorías: sin dolor, dolor sólo en relación a actividad física, dolor con actividades de la vida diaria (avd) menos de 2 veces por semana y dolor 2 o más veces a la semana con avd. Se realizó una evaluación antropométrica que incluía peso y talla. El examen físico fue realizado por un ortopedista infantil, e incluyó 
evaluación de la marcha (diferenciando 3 grupos: marcha convergente, neutra y divergente), evaluación de la alineación de las extremidades inferiores (varo, neutro y valgo) y podoscopía.

El análisis del perfil rotacional se realizó definiendo el tipo de marcha como neutra, convergente o divergente, según la posición adoptada por el pie al caminar en forma natural por un pasillo. La posición del pie fue medida en grados, según la marca dejada por el pie con respecto a una línea recta dibujada en el piso, la que representa el eje de avance de la marcha.

Luego se midió las rotaciones interna y externa de las caderas, para así estimar la ante o retroversión femoral según el método de Staheli. Finalmente se midió la torsión de la tibia, a través de la medición del ángulo muslo-pie, medición que pone al resto de las estructuras de la extremidad inferior en neutro para medir esta torsión en particular.

Se excluyeron 10 casos por presentar enfermedades neuromusculares (5 casos), secuelas de pie bot (3 casos), y pie cavo (2 casos).

El análisis de los datos se realizó con el software spss 11.0 (spss inc., Chicago, il), utilizando test de $\chi^{2}$ para variables discretas, coeficiente de correlación de pearson, y t-student para variables continuas, considerando un $\mathrm{p}<0,05$ como estadísticamente significativo.

\section{Resultados}

Un 53\% de los niños era de sexo masculino.
El promedio de edad fue de 7,1 años. La distribución por grupos de edad se muestra en la figura 1.

El análisis del tipo de marcha mostró la siguiente distribución: 58\% neutro, 23\% marcha divergente y $19 \%$ marcha convergente.

Los tres grupos presentaron una prevalencia de síntomas muy similar, alrededor de 5\% de dolor de pies relacionado con la actividad física, menos de 2 veces por semana y menos del $1 \%$ más de 2 veces por semana $(\mathrm{p}=0,234)$.

La edad tiene una relación muy estrecha con tipo de alineación, mostrando que la marcha convergente es muy prevalente en las etapas tempranas, para luego hacerse divergente en los niños mayores (figura 2).

La mayor rotación interna de las caderas, en el grupo convergente fue de $67^{\circ}$, comparado con el grupo de marcha neutra con $60^{\circ}$ y los con marcha divergente con $54^{\circ}(r=-0,1,0,049)$ (figura 3).

La torsión tibial, medida por el ángulo muslo-pie, tiene una relación directa con la edad y la marcha convergente $(\mathrm{r}=0,1, \mathrm{p}=0,045)$ (figura 4).

En el grupo de pacientes que cayeron en el rango de obesidad, la marcha es significativamente mas divergente $(p=0,035)$, que en el grupo eutrófico, pero no presentan más síntomas. Lo que se explicaría por la mayor rotación externa de las caderas.

El tipo de marcha no se correlacionó con la presencia de pie plano ni pie aducto $(\mathrm{p}>0,05)$.

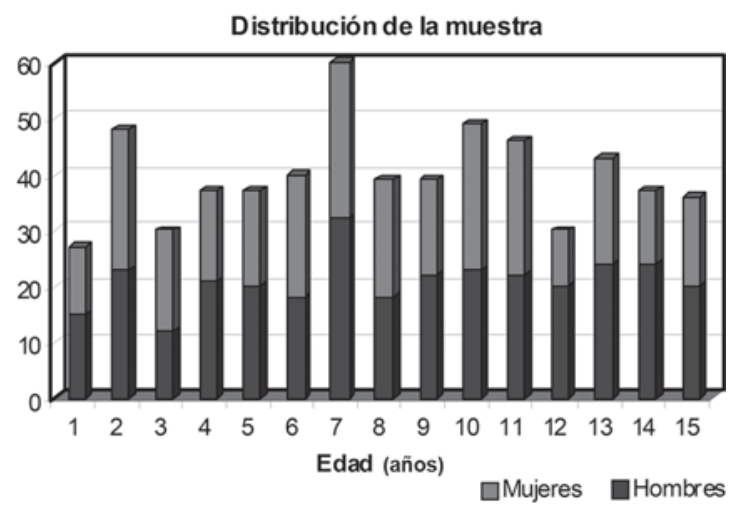

Figura 1. Distribución por sexo y edad. Estadístico utilizado: Prueba T Student para muestras independientes. $\mathrm{p}=0,103$.

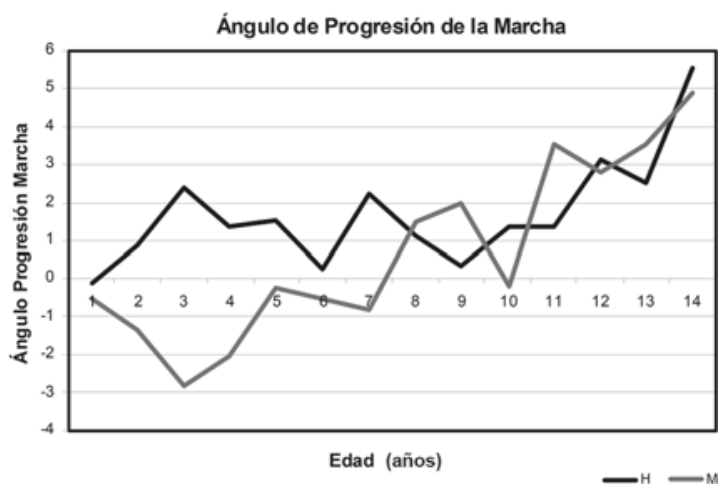

Figura 2. Evolución del ángulo de progresión del pie en las distintas edades. 


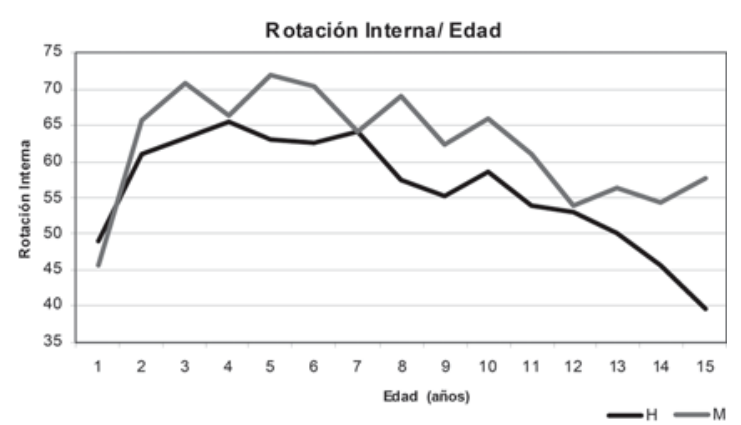

Figura 3. Evolución de la rotación interna de las caderas en las distintas edades. ${ }^{*} \mathrm{H}$ : hombres / M: mujeres. Estadístico utilizado: Coeficiente de Correlación de Pearson $(\mathrm{R}=-0,1$, $\mathrm{P}$ $=0,049)$.

\section{Discusión}

En nuestro estudio se demostró que la marcha convergente es un problema frecuente, pero en el caso de la mayoría de los niños examinados, no había motivado visitar al traumatólogo $(<1 \%)$ ni la presencia de síntomas había sido motivo de preocupación para los padres.

La mayoría de los niños evaluados: 58\% deambula en forma neutra, y los restantes se distribuyen en forma muy similar en marcha convergente y divergente.

Al realizar un análisis de todos los parámetros medidos que influencian el tipo de marcha, podemos observar las siguientes relaciones: se observó una fuerte correlación de la alineación de la marcha con la rotación interna de las caderas, lo que tiene que ver con la anteversión femoral, es decir, mientras más rotada hacia anterior está la cadera, mayor es la tendencia a la marcha convergente, lo que concuerda con estudios clásicos ${ }^{14-16}$. No sucede lo mismo con la rotación externa de caderas, por lo que no podría relacionarse la retroversión femoral con el tipo de marcha, o existen otras estructuras, más bien blandas, que influyen en la rotación externa de caderas.

Es así como la rotación interna de las caderas describe una curva similar a la marcha convergente: tiene un peak alrededor de los 2 a 3 años, momento en que ya ha cedido la contractura en rotación externa propia del recién

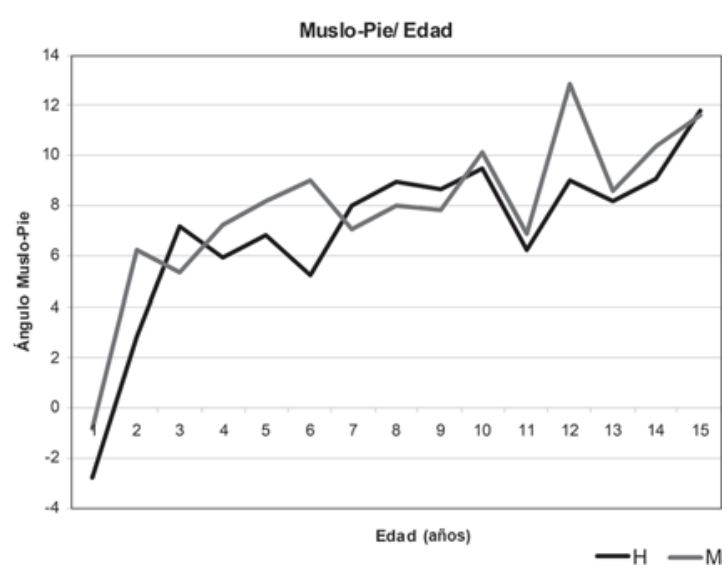

Figura 4. Evolución del ángulo muslo-pie (torsión tibial) en las distintas edades. Estadístico: Coeficiente de Correlación de Pearson ( $\mathrm{R}=0,1, \mathrm{p}=0,045)$.

nacido y va disminuyendo posteriormente lo que concuerda con lo descrito en la literatura ${ }^{12}$.

La torsión interna de la tibia también se correlaciona con el tipo de marcha, lo que sugiere que la tibia aporta también rotación al caminar.

En cuanto al rol en nuestra muestra del metatarso aducto ${ }^{17}$, la incidencia es tan pequeña (menor al 1\%), que no permite sacar conclusiones.

\section{Conclusiones}

Lo observado en nuestro trabajo, apoya el método descrito por Staheli para medición clínica de las distintas estructuras que influencian la marcha.

Se demuestra que existen características anatómicas de los huesos de las extremidades inferiores, que explican la rotación del pie al caminar, y que existe entre ellas, una compleja interacción, lo que asociado a la influencia de las partes blandas, son materia de estudio aún.

El médico se ve enfrentado a un gran desafío al encontrarse en la consulta con una alteración rotacional de las extremidades inferiores de un niño. El debe dar a conocer a los familiares las características fisiológicas del desarrollo de las extremidades inferiores en la infancia en forma clara y convincente, ya que está escrito en nuestra cultura el uso de "aparatos" para 
ayudar al buen desarrollo de las extremidades de los niños.

Además, cabe destacar que el desempeño deportivo de los niños que presentan condiciones como la marcha convergente o divergente, es exactamente el mismo que los con marcha neutra ${ }^{18,19}$.

Por otro lado, es conocido que ni la anteversión femoral ni la torsión tibial dejan secuelas para el futuro ${ }^{20}$. De esta manera evitaremos pedir exámenes innecesarios y realizar tratamiento a condiciones conocidamente fisiológicas.

\section{Referencias}

1.- Tachdjian's pediatric orthopedics. Third edition 2002; 63-82.

2.- Kakihana W, Akai M, Yamasaki N, Takashim T, Nakazawa K: Changes of joint moments in the gait of normal subjects wearing laterally wedged insoles. Am J Physical Med Rehabilit 2004; 83 (4): 273-8.

3.- Driano A, Staheli L, Staheli L: Psychosocial development and corrective shoewear use in childhood. J Pediatric Orthopedics 1998; 18 (3): 346-9.

4.- Fuchs $R$, Staheli L: Sprinting and intoeing. J Pediatric Orthopedics 1996; 16 (4): 489-91.

5.- Redmond AC: An evaluation of the use of gait plate inlays in the short-term management of the intoeing child. Foot \& Ankle International 1998; 19 (3): 1448.

6.- Knittel G, Staheli LT: The effectiveness of shoe modifications for intoeing. Orthop Clin North Am 1976; 7: 1019-25.

7.- Staheli LT: Editorial: normative data in pediatric orthopedics. J Pediatric Orthopedics 1996; 16 (5): 561-2.
8.- Staheli LT: Rotational problems in children. J Bone Joint Surg Am 1993; 75-a (6): 939-49.

9.- Staheli L, Corbett $M$ : Lower extremities rotational problems in children. J Bone Joint Surg 1985; 67 a: 39.

10.- Bastin JP: Pa-c lower extremity rotational disorders in children. Physician Assistant 2001; 25 (11): 30-3, 36-7.

11.- Jacquemier M, Jouve JL, Jimeno MT, Ramaherisson $P$, Giusiano B, Bollini G: Lower limb aspect: clinical evaluation in 1401 children. J Bone Joint Surg Br 1999; 81-b supplement iii: 355.

12.- Hunt D, Breach C, Friskin I: The combination of rotational abnormalities in children. 1998; 80-b (3s) supplement iii: 256.

13.- Staheli LT, Clawson DK, Hubbard DD: Medial femoral torsion: experience with operative treatment. Clin Orthop 1980; 146: 222-5.

14.- Heinrich SD, Sharps C: Lower extremity torsional deformities in children: a prospective comparison of two treatment modalities. Orthop Trans 1989; 13: 554-5.

15.- Shands AR, Steele MK: Torsion of the femur. A followup report on the use of the dunlap method for its determination. J Bone Joint Surg 1958; 40-a: 803-16.

16.- Fabry G, Macewen GD, Shands AR: Torsion of the femur. A follow-up study in normal and abnormal conditions. J Bone Joint Surg 1973; 55-a: 1726-38.

17.- Ponseti IV, Becker JR: Congenital metatarsus adductus: the results of treatment. J Bone Joint Surg 1966; 48a: $02-711$.

18.- Staheli LT, Lippert K, Denotter P: Femoral anteversion and physical performance in adolescent and adult life. Clin Orthop 1977; 129: 213-6.

19.- Farsetti, P, Weinstein SL, Ponseti IV: The long-term functional and radiographic outcomes of untreated and non-operatively treated metatarsus adductus. J Bone Joint Surg Am 1994; 76-a (2): 257-65.

20.- Tonnis D, Heinecke A: Acetabular and femoral anteversion: relationship with osteoarthritis of the hip. J Bone Joint Surg Am 1999; 81-a (12): 1747-70. 